\title{
Erratum
}

Journal of Microbiology (2012) Vol. 50, No. 4, pp. 613-617

DOI 10.1007/s12275-012-1596-5

\section{Lactobacillus delbrueckii ssp. lactis R4 Prevents Salmonella typhimurium SL1344-Induced Damage to Tight Junctions and Adherens Junctions}

Qinghua Yu, Liqi Zhu, Zhisheng Wang, Pengcheng Li, and Qian Yang*

Nanjing Agricultural University, Weigang 1, Nanjing, Jiangsu 210095, P.R. China

In the article by Yu et al. published in Journal of Microbiology 2012; 50, 613-617, the figure 1 is unfortunately incorrect. The figure 1 should be corrected as below. The text of the figure legends and the article remains unchanged as they are correct.

We apologize for any inconvenience that this may have caused.

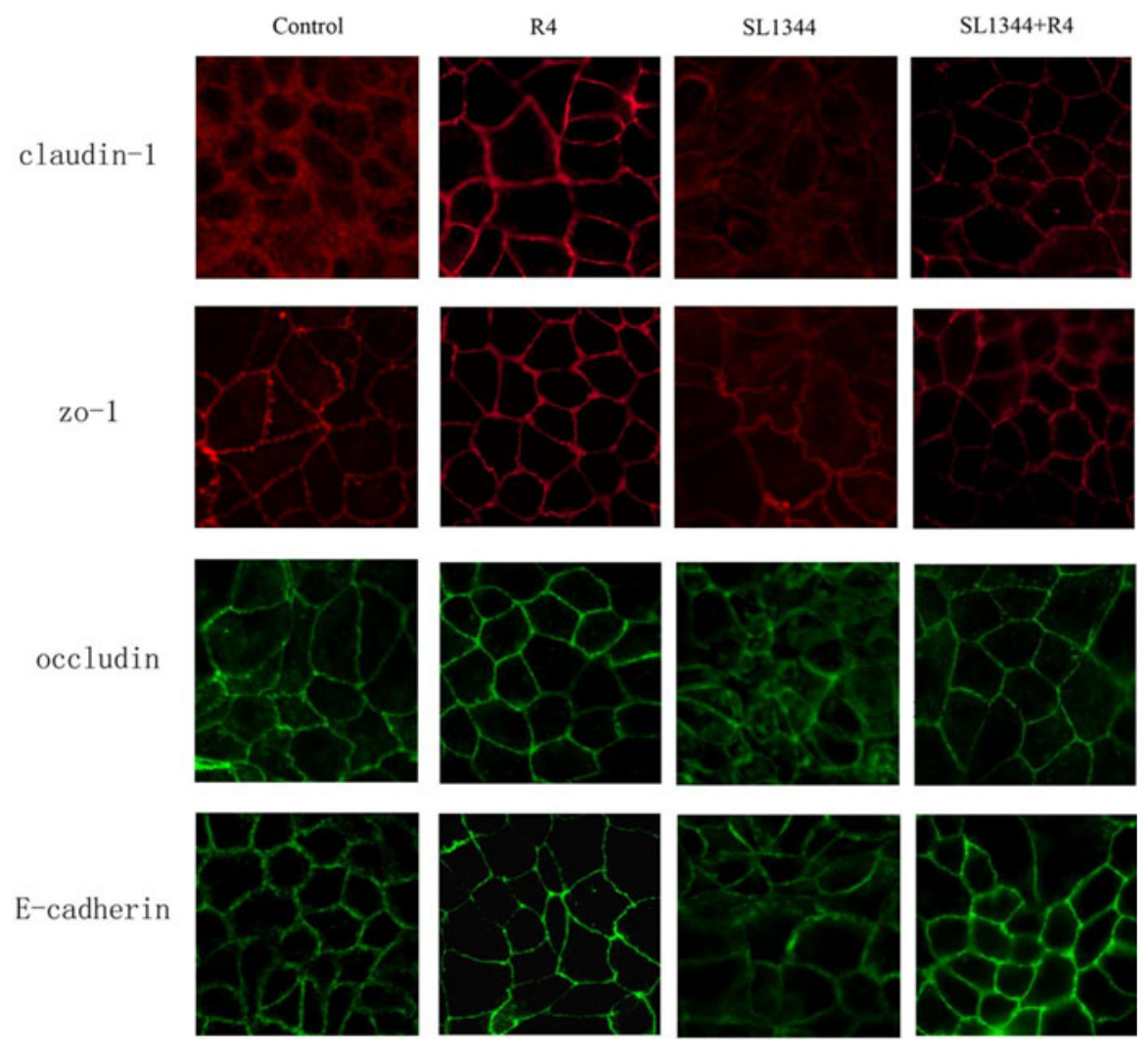

